\title{
O uso do propranolol na terapêutica do hemangioma infantil
}

The use of propranolol in child hemangioma therapy

O uso de propranolol em el tratamiento del hemangioma infantil

Vitoria Vilas Boas da Silva Bomfim ORCID: https://orcid.org/0000-0003-4897-0279

Centro Universitário Jorge Amado, Brasil E-mail: pesquisaclinica9@gmail.com

Maria Eduarda Lopes de Macedo Bezerra ORCID: https://orcid.org/0000-0001-6741-3190 Faculdade Estácio Rio Grande do Norte, Brasil E-mail: eduardalopes022@gmail.com

Maria Clara Teles Cabanelas Macedo ORCID: https://orcid.org/0000-0003-2025-7789 Universidade Salvador, Brasil E-mail: claracabanelas@gmail.com

Mikaele Montalvão Galliza Lima ORCID: https://orcid.org/0000-0001-9955-7914 Universidade Salvador, Brasil E-mail: mikaelegalliza@hotmail.com Hanah Passos Carneiro ORCID: https://orcid.org/0000-0002-8696-0146 Universidade Salvador, Brasil

E-mail: hanahpassos@yahoo.com.br

Emily da Silva Eberhardt

ORCID: https://orcid.org/0000-0003-2736-5686 Centro Universitário Cenecista de Osório, Brasil

E-mail:milyeberhardt@gmail.com

Mirella Figueirôa Vieira

ORCID: https://orcid.org/0000-0002-4230-143X Universidade Tiradentes, Brasil

E-mail: mirellaalencar2015@gmail.com

Monalisa Batatinha de Castro Silva ORCID: https://orcid.org/0000-0001-9096-9242 Universidade Estadual da Bahia Campus VII, Brasil

E-mail: monalisabcs89@gmail.com

Lorenna Evellyn Pereira de Paula ORCID: https://orcid.org/0000-0002-4885-2427

Universidade Tiradentes, Brasil

E-mail: lorenna_ev@hotmail.com

Vyttória Oliveira Albuquerque ORCID: https://orcid.org/0000-0001-7242-4118 Centro Universitário Tiradentes, Brasil

E-mail: vyttoriaalbuquerque@hotmail.com

Ayana Cavalcante da Paz ORCID: https://orcid.org/0000-0003-1304-5091

Centro Universitário Uninovafapi, Brasil

E-mail: ayanapazcavalcante@gmail.com

Lúcio Petterson Tôrres da Silva ORCID: https://orcid.org/0000-0002-8423-0473 Centro Universitário do Vale do Ipojuca, Brasil E-mail: petterson17@gmail.com

Erica Williams de Moreira Lima ORCID: https://orcid.org/0000-0003-3957-5699

Centro Universitário Uninovafapi, Brasil

E-mail: williams-erica1992@outlook.com

Maria Aparecida Guimarães da Silva ORCID: https://orcid.org/0000-0001-7364-1173

Universidade Maurício de Nassau, Brasil E-mail: enfa.aguimaraes@gmail.com 


\begin{abstract}
Resumo
Objetivo: Avaliar o uso do propranolol como opção terapêutica para o hemangioma infantil, descrevendo as evidências encontradas na literatura, acerca das vantagens desta nova terapêutica quando comparada às terapias convencionais. Métodos: Trata-se de uma revisão integrativa de literatura, com artigos nas bases de dados Biblioteca Virtual de Saúde (BVS) e PubMed, nos idiomas português e inglês, utilizando os descritores "propranolol treatment","propranolol","infantile hemangioma" e "hemangioma of infancy". Resultados: Foram encontrados na busca de literatura 1876 artigos, nos quais após a aplicação dos critérios de inclusão e exclusão e a revisão em pares ficaram 18 artigos a serem lidos na íntegra. Dos 18 artigos, foram incluídos 1380 pacientes que preencheram os critérios de inclusão. Um total de $56.5 \%$ eram do sexo feminino e compreendiam o intervalo de idade de 1 semana de vida a 9 anos de idade. Considerações Finais: O propranolol é considerado uma terapia alternativa para o tratamento do hemangioma infantil por apresentar uma eficácia elevada e menos efeitos adversos.
\end{abstract}

Palavras-chave: Terapêutica; Propranolol; Hemangioma; Criança.

\begin{abstract}
Objective: To evaluate the use of propranolol as a therapeutic option for childhood hemangioma, describing the evidence found in the literature, about the advantages of this new therapy when compared to conventional therapies. Methods: This is a integrative literature review, with articles in the Virtual Health Library (VHL) and PubMed databases, in Portuguese and English, using the keywords "propranolol treatment", "propranolol", "infantile hemangioma" and "hemangioma of infancy". Results: A total of 1876 articles were found in the literature search, in which, after applying the inclusion and exclusion criteria and the peer review, there were 18 articles to be read in full. Of the 18 articles, 1380 patients who met the inclusion criteria were included. A total of 56.5\% were female and comprised the age range from 1 week of life to 9 years of age. Final considerations: Propranolol is considered an alternative therapy for the treatment of infantile hemangioma because of its high efficacy and less adverse effects.
\end{abstract}

Keywords: Therapeutics; Propranolol; Hemangioma; Child.

\title{
Resumen
}

Objetivo: Evaluar el uso de propranolol como opción terapéutica para el hemangioma infantil, describiendo la evidencia encontrada en la literatura, sobre las ventajas de esta nueva terapia en comparación con las terapias convencionales. Métodos: Se trata de una revisión bibliográfica inegrativa, con artículos en la Biblioteca Virtual en Salud (BVS) y bases de datos PubMed, en portugués e inglés, utilizando las palabras clave "tratamiento con propranolol", "propranolol", "hemangioma infantil" y "hemangioma de la infancia". Resultados: Se encontraron un total de 1876 artículos en la búsqueda bibliográfica, en los cuales, luego de aplicar los criterios de inclusión y exclusión y la revisión por pares, hubo 18 artículos para ser leídos en su totalidad. De los 18 artículos, se incluyeron 1380 pacientes que cumplieron con los criterios de inclusión. El 56,5\% eran mujeres y comprendían el rango de edad desde 1 semana de vida hasta 9 años de edad. Consideraciones finales: El propranolol se considera una terapia alternativa para el tratamiento del hemangioma infantil debido a su alta eficacia y menos efectos adversos.

Palabras clave: Terapéutica; Propranolol; Hemangioma; Niño.

\section{Introdução}

Os hemangiomas infantis (HI) resultam de alterações do processo de angiogênese e constituem-se como uma rápida proliferação de células endoteliais, formando tumores vasculares que surgem nas primeiras semanas de vida. São classificados em: superficiais, consistindo em placas ou tumorações de cor vermelho-vivo; profundos, havendo formação de nódulos elevados, geralmente da cor da pele ou azulados; mistos, quando coexistem os componentes dos dois subtipos (Kwon, Seefeldt \& Drolet, 2013; Campos Júnior, Burns \& Lopez, 2014). Considerado o tumor mais comum da infância, a incidência do hemangiomas infantisI varia em torno de 5 a $10 \%$ e acomete principalmente a etnia branca, sendo mais prevalente no sexo feminino (2,4 a 4:1), atingindo principalmente cabeça e pescoço (Szychta, Stewart \& Anderson, 2014; Wedgeworth, Glover, Irvine, Neri, Baselga, Clayton \& Flohr, 2016).

Apesar de ser classificado como um tumor benigno e na maioria dos casos não necessitar de intervenção médica, aproximadamente $20 \%$ das crianças com hemangiomas infantis precisam de cuidados médicos, devido às complicações com ulceração, comprometimento visual e das vias aéreas, além de cicatrizes residuais e desfiguração (Wedgeworth et al., 2016; Buckmiller, Munson, Dyamenahalli, Dai \& Richter, 2010). 
Research, Society and Development, v. 10, n. 8, e10010816968, 2021

(CC BY 4.0) | ISSN 2525-3409 | DOI: http://dx.doi.org/10.33448/rsd-v10i8.16968

A patogênese desses tumores não é completamente conhecida. Estudos avaliando espécimes patológicos demonstraram haver expressão aumentada do transportador de glicose GLUT-1 em células 379 Protocolos Clínicos e Diretrizes Terapêuticas endoteliais do hemangioma bem como de antígenos vasculares associados à placenta. Tais alterações não são observadas em células endoteliais normais, podendo ser consideradas um marcador fenotípico do hemangioma. Fatores celulares e moleculares, tais como fator de crescimento vascular endotelial, fator de crescimento de fibroblastos e fator de crescimento semelhante à insulina tipo 2, estão relacionados com as fases de proliferação e regressão (BRASIL, 2013)

$\mathrm{O}$ tratamento dos casos complicados de hemangiomas infantis era feito, tradicionalmente, através de terapia sistêmica com corticosteroides, sendo a primeira escolha o corticosteróide tópico, seguido da terapia com interferon-alfa, vincristina, laser e cirurgia. No entanto, vários são os efeitos colaterais observados com o uso destas abordagens terapêuticas, como diplegia espástica (decorrente da ação do interferon) além das, já conhecidas, repercussões clínicas do uso contínuo de corticosteróide sistêmico, a saber, imunossupressão, fácies cushingoide, supressão da adrenal e hipertensão, gerando muitas vezes a não evidenciação de benefícios (Tan, Itinteang \& Leadbitter, 2011; Li, Xu, Gao \& Huo, 2015; Yun, Gyon, Yang, Lee, Park \& Park, 2015).

O uso do propranolol como opção terapêutica para o hemangioma infantil surgiu através de uma descoberta acidental em 2008, quando foi notado melhora nas lesões em dois pacientes pediátricos com cardiopatia, ambos em uso desta droga. A partir de então, vários casos foram sendo documentados, a fim de tentar estabelecer a ação do propranolol sobre esse tumor vascular. Um estudo envolvendo hemangiomas segmentares e de acometimento periorbital e das vias aéreas, a nova terapêutica apresentou inibição da proliferação do hemangioma, com menos de 48 horas do início do tratamento em $74 \%$ dos pacientes (Kwon et al., 2013; Junior \& Burns, 2014; Szychta, Stewart \& Anderson, 2014).

Deste modo, a terapia com propranolol parece ser a melhor opção terapêutica, haja vista os efeitos colaterais dos corticosteróides. Contudo, a descrição dos mecanismos de ação do propranolol no controle do crescimento do hemangioma e sua regressão ainda não é totalmente conhecido, mas acredita-se que seu efeito vasoconstrictor age diminuindo a expressão de fatores de crescimento endotelial (VEGF) e fator de crescimento fibroblástico intervindo no processo de angiogênese (Junior \& Burns, 2014; Li et al., 2015; Yun, Gyon, Yang, Lee, Park \& Park, 2015).

Ainda buscando avaliar a interação da droga sobre o hemangioma infantil, um estudo realizado com 106 crianças, em uso de propranolol para tratamento de casos complicados de hemangioma infantil, chegou ao resultado de $97 \%$ da amostra total apresentando regressão do hemangioma. A involução das tumorações, com redução significativa no tamanho e clareamento da coloração, foi registrada através de fotografias ao longo do tratamento (Li et al., 2015).

Por ser um beta-bloqueador não seletivo, os efeitos adversos do propranolol não foram totalmente esclarecidos, no entanto não se deve usar em casos de anormalidade nos indicadores da pressão arterial e frequência cardíaca, além de alterações nos índices glicêmicos. Além disso, seu uso é contra-indicado na Síndrome PHACES, na qual pode haver risco de acidente vascular isquêmico. Ademais, devido a grande aderência ao uso do propranolol como droga de escolha para tratar o hemangioma infantil, começou-se a se usar também a apresentação tópica do propranolol, nos casos de hemangiomas superficiais (Kwon et al., 2013; Buckmiller et al., 2011; Li et al., 2015).

Hemangiomas complicados requerem a intervenção precoce, através da utilização de terapêutica eficaz e segura para o controle do crescimento de massa tumoral, sendo determinante para prevenir a extensão de lesões e o risco de desfiguração. $\mathrm{O}$ uso do propranolol tem sido amplamente empregado nos casos graves, obtendo-se resposta rápida, necessitando, no entanto, de maiores esclarecimentos quanto a seu potencial efeito terapêutico e restrições, quando comparado a terapias tradicionais (Jing et al., 2016).

Descrever o que tem sido constatado sobre o propranolol como nova opção terapêutica ao hemangioma infantil possibilita, portanto, analisar seu impacto nas perspectivas futuras de tratamento deste tumor vascular. O presente estudo visa 
compreender melhor a repercussão do uso do propranolol, enquanto terapia recém descoberta para o tratamento de hemangioma infantil.

\section{Metodologia}

Trata-se de uma revisão integrativa de literatura, este tipo de estudo se dá por meio de seis etapas sendo elas elaboração da pergunta norteadora, busca na literatura científica, coleta de dados dos artigos escolhidos, análise crítica e reflexiva dos estudos selecionados, discussão dos resultados e apresentação em forma de revisão integrativa (Souza, Silva \& Carvalho, 2010).

Foram realizadas buscas nas bibliotecas de dados Biblioteca Virtual de Saúde (BVS), Biblioteca Regional de Medicina (BIREME) e U.S. National Library of Medicine (PubMed), com o objetivo de selecionar artigos, originais, nos idiomas inglês e português que foram publicados entre o período de janeiro de 2010 e maio de 2020, que abordem o uso do propranolol no tratamento do hemangioma infantil.

Tal período de pesquisa se justifica, na medida em que se busca um levantamento mais atual da literatura. para o levantamento bibliográfico serão utilizadas as combinações dos descritores "propranolol treatment", "propranolol”, "infantile hemangioma" e "hemangioma of infancy", através da seguinte equação booleana: ("propranolol treatment" OR "propranolol” AND "infantile hemangioma" OR "hemangioma of infancy").

Após obtidos os artigos resultantes, foi realizada uma seleção inicial, tendo por base a leitura dos títulos e resumos. Posteriormente será feita a leitura integral e serão elegidos os que se demonstrarem condizentes com o tema do presente estudo por meio de revisão por pares, a fim de escolher os artigos relevantes para realizar a análise dos resultados.

Foram incluídos na seleção artigos, em idiomas ingles e portugues, que foram publicados no recorte temporal previamente estabelecido e que discorrem sobre as implicações do uso terapêutico do propranolol em crianças diagnosticadas com hemangioma infantil. Os tipos de estudo selecionados foram: estudos observacionais, estudos transversais, estudos de coorte e ensaios clínicos. Também fizeram parte da seleção estudos que estejam nas referências dos artigos selecionado, que forem considerados relevantes ao tema em estudo. Serão desconsiderados artigos que abordam o uso de propranolol na terapêutica de outros tipos de hemangiomas, que não o hemangioma infantil.

Foram excluídas também revisões sistemáticas e não sistemáticas, relatos de caso, estudos em idiomas diferentes dos supracitados e que tenham sido publicados fora do período especificado. Por não se tratar de pesquisa envolvendo seres humanos, este estudo não necessita de parecer do Comitê de Ética em Pesquisa. Todavia, as autoras comprometem-se manter a integridade das informações contida nos artigos selecionados, bem como, a citar todas as fontes utilizadas na construção deste documento.

\section{Resultados}

Foram encontrados na busca de literatura 1876 artigos (108 BVS e 1768 PubMed), nos quais após a aplicação dos critérios de inclusão e exclusão restaram 76 artigos (9 BVS e 67 PubMed) para a revisão em pares. Após a revisão em pares ficaram 18 artigos a serem lidos na íntegra. Dos 18 artigos, foram incluídos 1380 pacientes que preencheram os critérios de inclusão. Um total de $56.5 \%$ eram do sexo feminino e compreendiam o intervalo de idade de 1 semana de vida a 9 anos de idade, conforme a Quadro 1. 
Quadro 1 - Dados das intervenções avaliadas nos estudos selecionados.

\begin{tabular}{|c|c|c|c|c|}
\hline ESTUDO & $\begin{array}{l}\text { TIPO DE } \\
\text { ESTUDO }\end{array}$ & $\begin{array}{c}\text { NÚMERO DE } \\
\text { PARTICIPANTES }\end{array}$ & $\begin{array}{l}\text { MEDICAÇÕES } \\
\text { E TEMPO DE } \\
\text { DURAÇÃO }\end{array}$ & DESFECHO \\
\hline 1 & $\begin{array}{l}\text { Ensaio Clinico } \\
\text { Randomizado }\end{array}$ & $\begin{array}{c}40 \text { crianças (9 } \\
\text { semanas- } 5 \text { anos) }\end{array}$ & $\begin{array}{l}\text { Propranolol } \mathrm{x} \\
\text { placebo por } 6 \\
\text { meses }\end{array}$ & $\begin{array}{l}\text { Em } 4 \text { semanas, o cloridrato de } \\
\text { propranolol foi mais eficaz no } \\
\text { tratamento, reduzindo o HI. }\end{array}$ \\
\hline 2 & $\begin{array}{l}\text { Ensaio Clinico } \\
\text { Randomizado }\end{array}$ & $\begin{array}{c}19 \text { crianças ( } 2 \\
\text { semanas- } 6 \text { meses) }\end{array}$ & $\begin{array}{l}\text { Propranolol } \mathrm{x} \\
\text { predinisolona por } 4 \\
\text { meses }\end{array}$ & $\begin{array}{l}\text { A predinisolona e o propranolol não } \\
\text { diferiu nos resultados finais do } \\
\text { tratamento. Só que o propranolol } \\
\text { apresentou menor efeito adverso. }\end{array}$ \\
\hline 3 & $\begin{array}{c}\text { Estudo } \\
\text { Retrospectivo }\end{array}$ & $\begin{array}{l}89 \text { crianças ( } 1 \text { mês - } \\
1 \text { ano e } 6 \text { meses) }\end{array}$ & $\begin{array}{l}\text { Propranolol } \mathrm{x} \\
\text { maleato de timolol } \\
0,5 \% \text { por } 9 \text { meses }\end{array}$ & $\begin{array}{l}\text { O propranolol oral combinado com o } \\
\text { tratamento de timolol tópico é muito } \\
\text { eficaz para o tratamento do HI, e pode } \\
\text { ser utilizado para o tratamento de } \\
\text { primeira linha. }\end{array}$ \\
\hline 4 & $\begin{array}{c}\text { Estudo } \\
\text { Retrospectivo }\end{array}$ & $\begin{array}{l}42 \text { crianças ( } 2 \text { meses } \\
-5 \text { meses })\end{array}$ & $\begin{array}{l}\text { Propranolol } \mathrm{x} \\
\text { predinisolona }+ \\
\text { triancinolona por } \\
\quad 51 \text { meses }\end{array}$ & $\begin{array}{c}\text { O propranolol deve ser considerado } \\
\text { como terapia de primeira linha ou } \\
\text { como parte da abordagem } \\
\text { multidisciplinar. }\end{array}$ \\
\hline 5 & $\begin{array}{c}\text { Estudo } \\
\text { Retrospectivo }\end{array}$ & $\begin{array}{l}89 \text { crianças ( } 1 \text { mês - } \\
1 \text { ano e } 5 \text { meses) }\end{array}$ & $\begin{array}{l}\text { Propranolol } \mathrm{x} \\
\text { timolol tópico por } \\
48 \text { meses }\end{array}$ & $\begin{array}{l}\text { O propranolol oral combinado com o } \\
\text { tratamento tópico com timolol é muito } \\
\text { eficaz e seguro para os compostos HI. }\end{array}$ \\
\hline 6 & Estudo de Coorte & $\begin{array}{c}58 \text { crianças ( } 3 \text { meses } \\
-7 \text { meses) }\end{array}$ & $\begin{array}{l}\text { Propranolol } \mathrm{x} \\
\text { placebo por } 7 \\
\text { meses }\end{array}$ & $\begin{array}{c}\text { Observou-se que pacientes tratados } \\
\text { com propranolol isolado foram menos } \\
\text { propensos a serem submetidos a } \\
\text { tratamento invasivo. }\end{array}$ \\
\hline 7 & $\begin{array}{l}\text { Ensaio Clinico } \\
\text { Randomizado }\end{array}$ & $\begin{array}{c}460 \text { crianças ( } 1 \text { mês- } \\
5 \text { meses })\end{array}$ & $\begin{array}{l}\text { Propranolol x } \\
\text { placebo por } 24 \\
\text { semanas }\end{array}$ & $\begin{array}{c}\text { O estudo mostrou que o uso de } \\
3 \mathrm{mg} / \mathrm{kg} \text { de propranolol por dia durante } \\
6 \text { meses de tratamento do HI foi eficaz }\end{array}$ \\
\hline 8 & $\begin{array}{l}\text { Ensaio Clinico } \\
\text { Randomizado }\end{array}$ & $\begin{array}{c}23 \text { crianças ( } 1 \text { mês - } \\
15 \text { meses) }\end{array}$ & $\begin{array}{l}\text { Propranolol } \mathrm{x} \\
\text { atenolol por } 6 \\
\text { meses }\end{array}$ & $\begin{array}{c}\text { O estudo mostrou que o atenolol pode } \\
\text { ser tão eficaz quanto o propranolol. } \\
\text { Não houve diferenças significativas } \\
\text { entre os resultados. }\end{array}$ \\
\hline 9 & $\begin{array}{l}\text { Ensaio Clinico } \\
\text { Randomizado }\end{array}$ & $\begin{array}{c}19 \text { crianças ( } 2 \text { meses } \\
\text { e meio- } 17 \text { meses })\end{array}$ & $\begin{array}{l}\text { Propranolol } \mathrm{x} \\
\text { terapia com laser } \\
\text { de corante (PDL) } \\
\text { por } 12 \text { semanas }\end{array}$ & $\begin{array}{l}\text { Foi possível observar que a } \\
\text { combinação do PDL e propranolol são } \\
\text { seguras e eficazes, tendo uma resposta } \\
\text { satisfatória maior que o uso isolado. }\end{array}$ \\
\hline 10 & $\begin{array}{l}\text { Ensaio Clinico } \\
\text { Randomizado }\end{array}$ & $\begin{array}{c}40 \text { crianças ( } 4 \\
\text { semanas }-8 \text { semanas) }\end{array}$ & $\begin{array}{l}\text { Propranolol } \mathrm{x} \\
\text { propranolol com } \\
\text { corticoide } \\
\text { sistêmico por } 6 \\
\text { meses }\end{array}$ & $\begin{array}{c}\text { Em caso de risco de vida a } \\
\text { combinação de propranolol e } \\
\text { corticoide pode ser uma ótima opção } \\
\text { pois oferece uma resposta de forma } \\
\text { rápida e eficaz. }\end{array}$ \\
\hline 11 & Estudo de Coorte & $\begin{array}{c}31 \text { crianças (1 mês - } \\
9 \text { anos) }\end{array}$ & $\begin{array}{l}\text { Propranolol } \mathrm{x} \\
\text { placebo por } 6 \\
\text { meses }\end{array}$ & $\begin{array}{l}\text { O uso de propranolol apresentou-se } \\
\text { eficaz tanto para o tratamento de } \mathrm{HI} \\
\text { quanto no declínio de }>20 \% \text { na } \\
\text { frequência cardíaca. }\end{array}$ \\
\hline 12 & $\begin{array}{l}\text { Ensaio Clinico } \\
\text { Randomizado }\end{array}$ & $\begin{array}{l}30 \text { crianças (1 semana } \\
-8 \text { meses })\end{array}$ & $\begin{array}{l}\text { Propranolol } \mathrm{x} \\
\text { predinisolona } \mathrm{x} \\
\text { propranolol com } \\
\text { predinisolona por } 3 \\
\text { meses }\end{array}$ & $\begin{array}{l}\text { O propranolol teve o efeito terapêutico } \\
\text { mais consistente e rápido que o da } \\
\text { predinisolona isolada e combinação } \\
\text { teve eficácia comparada ao } \\
\text { propranolol. }\end{array}$ \\
\hline
\end{tabular}


Research, Society and Development, v. 10, n. 8, e10010816968, 2021

(CC BY 4.0) | ISSN 2525-3409 | DOI: http://dx.doi.org/10.33448/rsd-v10i8.16968

\begin{tabular}{|c|c|c|c|c|}
\hline 13 & Estudo de Coorte & $\begin{array}{l}34 \text { crianças (0 dias - } \\
300 \text { dias) }\end{array}$ & $\begin{array}{c}\text { Propranolol } x \\
\text { terapia } \\
\text { intralesional com } \\
\text { corticosteroide por } \\
6 \text { meses }\end{array}$ & $\begin{array}{c}\text { A terapia intralesional se mostrou } \\
\text { bastante eficiente e segura e pode ser } \\
\text { considerada como opção quando a } \\
\text { terapia com propranolol não for } \\
\text { possível. }\end{array}$ \\
\hline 14 & $\begin{array}{c}\text { Estudo } \\
\text { Observacional }\end{array}$ & $\begin{array}{l}31 \text { crianças (0 dias - } \\
250 \text { dias })\end{array}$ & $\begin{array}{c}\text { Propranolol } \mathrm{x} \\
\text { predinisolona } \mathrm{x} \\
\text { propranolol com } \\
\text { predinisolona por } 3 \\
\text { meses }\end{array}$ & $\begin{array}{c}\text { O propranolol teve o efeito terapêutico } \\
\text { mais consistente e rápido que o da } \\
\text { predinisolona isolada e combinação } \\
\text { teve eficácia comparada ao } \\
\text { propranolol. }\end{array}$ \\
\hline 15 & $\begin{array}{l}\text { Ensaio Clínico } \\
\text { Randomizado }\end{array}$ & $\begin{array}{c}34 \text { crianças ( } 0 \text { dias- } 9 \\
\text { meses })\end{array}$ & $\begin{array}{l}\text { Propranolol } \mathrm{x} \\
\text { corticosteroides } \\
\text { por } 16 \text { semanas }\end{array}$ & $\begin{array}{l}\text { O propranolol foi tão eficaz quanto os } \\
\text { corticosteróides. }\end{array}$ \\
\hline 16 & Ensaio Clínico & $\begin{array}{l}45 \text { crianças ( } 35 \text { dias - } \\
150 \text { dias) }\end{array}$ & $\begin{array}{l}\text { Propranolol por } 6 \\
\text { meses }\end{array}$ & $\begin{array}{c}\text { Foi observado que o uso de } \\
\text { propranolol é seguro e eficaz e } \\
\text { apresenta sucesso quando } \\
\text { administrado até os } 12 \text { meses de vida. }\end{array}$ \\
\hline 17 & Ensaio Clínico & $\begin{array}{c}32 \text { crianças ( } 35 \text { dias - } \\
150 \text { dias) }\end{array}$ & $\begin{array}{l}\text { Propranolol por } 24 \\
\text { semanas }\end{array}$ & $\begin{array}{l}\text { Mostrou-se eficaz e seguro nas doses } \\
\text { de } 3 \mathrm{mg} / \mathrm{kg} / \mathrm{dia} \text {. }\end{array}$ \\
\hline 18 & Ensaio Clínico & $\begin{array}{c}264 \text { crianças ( } 2 \text { anos - } \\
6 \text { anos) }\end{array}$ & $\begin{array}{l}\text { Propranolol por } 4 \\
\text { meses }\end{array}$ & $\begin{array}{c}\text { O propranolol é seguro para o uso na } \\
\text { pediatria e traz eficácia no tratamento } \\
\text { de HI. }\end{array}$ \\
\hline
\end{tabular}

Fonte: Autores (2021).

Os estudos $1,2,7,8,9,10,12$ e 15 tratam-se de ensaios clínicos randomizados com um total de 665 participantes com idade entre 1 semana e 5 anos (Hogeling, Adams \& Wargon, 2011; Bauman, McCarter, Guzzetta, Shin, Oh, Preciado \& Puttgen, 2014; Léauté-Labrèze, Hoeger, Mazereeuw-Hautier, Guibaud, Baselga, Posiunas \& Voisard, 2015; Abarzúa-Araya, Navarrete-Dechent, Heusser, Retamal \& Zegpi-Trueba, 2014; Ehsani, Noormohammadpoor, Abdolreza, Balighi, Arianian \& Daklan, 2014; Aly, Hamza, Kader, Saafan, Ghazy \& Ragab, 2015 ; Malik, Menon, Rao \& Samujh, 2013; Kim, Choi, Choi, Park, Hong, Kim \& Jeong, 2017). Já os estudos 3, 4 e 5 tratam-se de estudos retrospectivos com 220 participantes no total, com idades que variam entre 1 mês e 1 ano e 6 meses, sendo que é possível observar a prevalência do sexo feminino em todos eles (Figura 1) (Ji, Chen, Xiang, Xu, Jiang, Liu \& Yang, 2016; Ge, Zheng, Zhang, Yuan \& Zhao, 2016).

Evidencia-se que o propranolol foi mais eficaz no tratamento envolvendo os hemangiomas em até 4 semanas em todos os estudos. Além disso, nos casos em que foi combinado com o timolol tópico a $0,5 \%$ também apresentou eficácia, diferente da prednisolona que causou o aumento do número de eventos adversos graves. Sendo assim, 152 crianças abandonaram os estudos por conta de infecções no trato respiratório superior, tendo como eventos adverso mais grave o óbito em 3 crianças. 
Research, Society and Development, v. 10, n. 8, e10010816968, 2021

(CC BY 4.0) | ISSN 2525-3409 | DOI: http://dx.doi.org/10.33448/rsd-v10i8.16968

Figura 1 - Tempo teste dos medicamentos concomitantes ao propranolol.

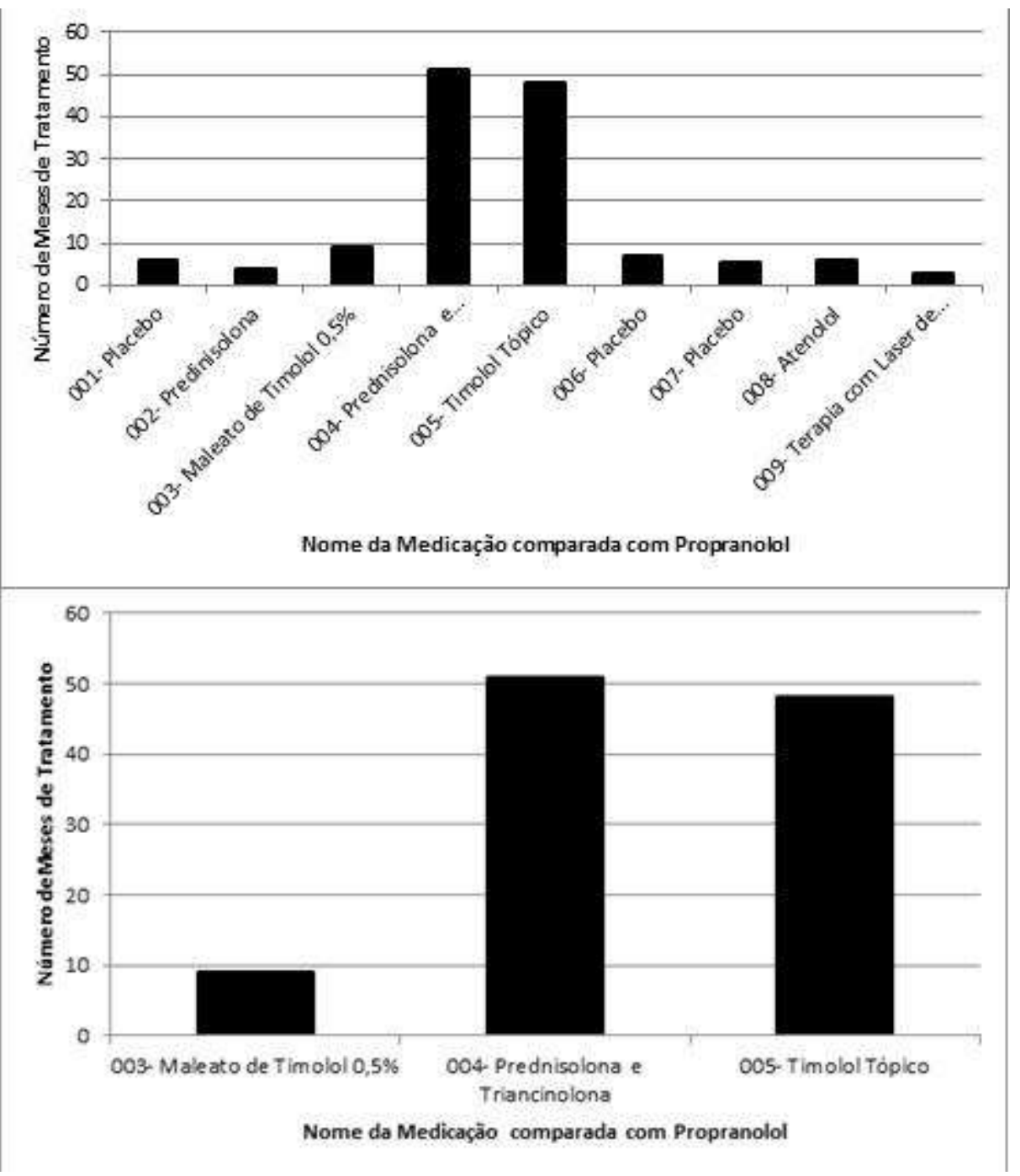

Fonte: Autores (2021).

Os estudos 6, 11 e 13 tratam-se de estudos de coorte com um total de 123 participantes, com idades entre 1 mês e 9 anos, já o estudo 14 trata-se de um estudo observacional com 31 participantes no total. Observa-se prevalência do sexo feminino em todos esses estudos (Figura 2) (Perkins, Chen, Saltzman, Manning \& Parikh, 2014; Sondhi \& Patnaik, 2013; Janmohamed, Madern, Nieuwenhuis, de Laat, \& Oranje, 2012; Holmes, Mishra, Gorst \& Liew, 2011).

Conclui-se que o propranolol foi mais eficaz no tratamento involuindo os hemangiomas em até $87 \%$ dos casos, sendo a droga de principal escolha e que o corticosteróide deve ser considerado nos casos em que o propranolol é contra indicado. Nesse caso, não houve eventos adversos. 
Research, Society and Development, v. 10, n. 8, e10010816968, 2021

(CC BY 4.0) | ISSN 2525-3409 | DOI: http://dx.doi.org/10.33448/rsd-v10i8.16968

Figura 2 - Tempo teste dos medicamentos concomitantes ao propranolol.
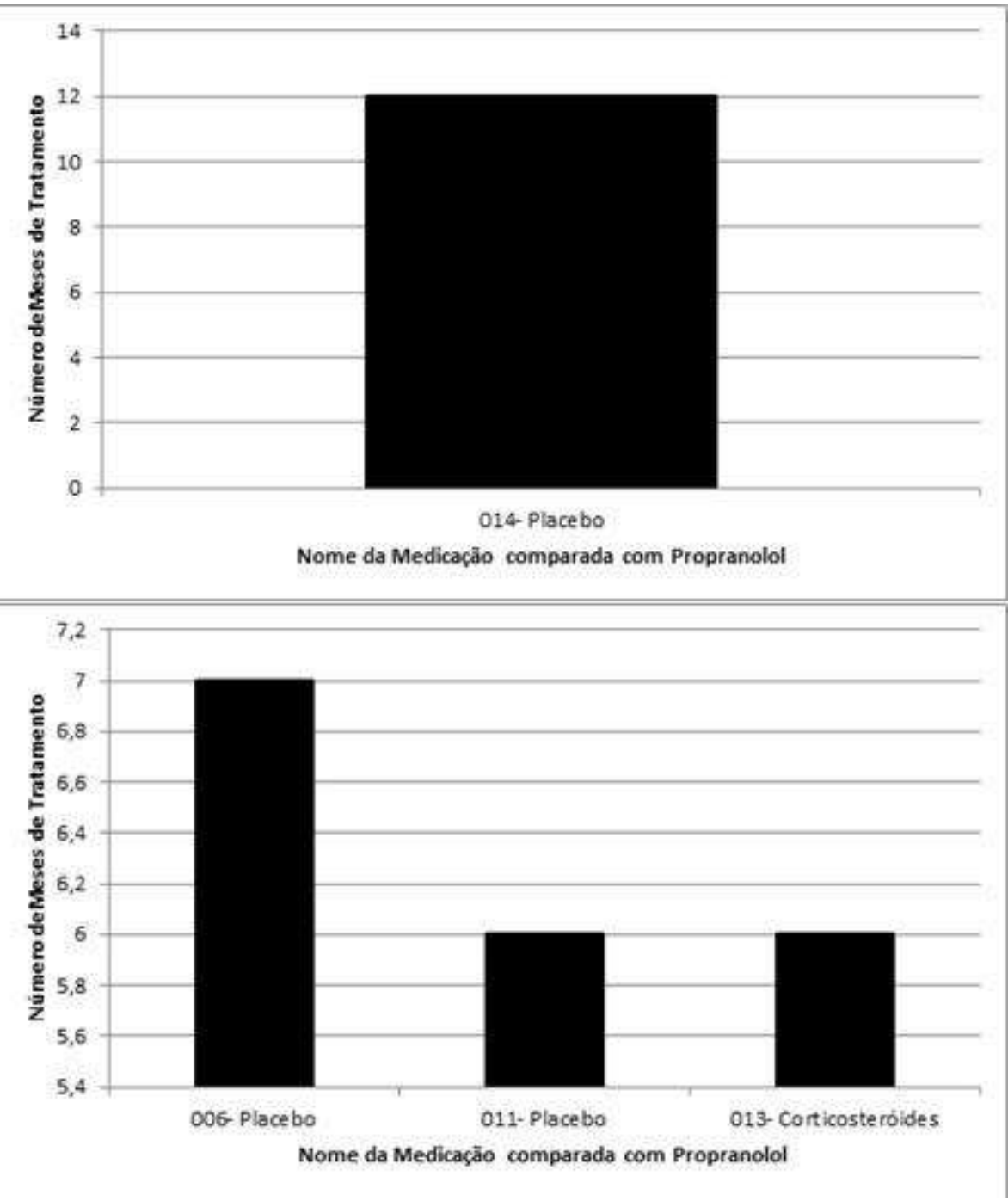

Fonte: Autores (2021).

Os estudos 16, 17 e 18 tratam-se de ensaios clínicos com 341 participantes no total entre 35 dias e 6 anos, houve prevalência do sexo feminino (Baselga, Dembowska-Baginska, Przewratil, González-Enseñat, Wyrzykowski, Torrelo \& Voisard, 2018; Kaneko, Sasaki, Baba, Koh, Matsui, Ohjimi \& Higuchi, 2017; Hu, Zhou, Huang, Chang, Qiu, Ma \& Li, 2016 ). Consoante a este estudo controlado, pode-se observar que diante da comparação desses 3 tipos de placebo e o propranolol, evidenciou-se que o primeiro estudo teve a duração de 6 meses, o segundo durou 5 meses e meio, e o terceiro, 4 meses. Além disso, notou-se a redução significativa do volume do hemangioma por meio do tratamento com o propranolol, em relação aos outros 3 tipos de fármacos testados (Figura 3).

Conclui-se que o propranolol é a droga de escolha mais segura e mais eficaz contra os casos de hemangioma infantil e que não houveram eventos adversos. Dessa forma, comparado a outras terapias, o tratamento utilizando o propranolol demonstrou ter uma eficácia igual ou maior que o uso de outras medicações, apresentando também ter menos efeitos adversos, sendo assim uma opção mais acessível e eficaz na terapêutica do hemangioma infantil. 
Figura 3 - Tempo Teste dos medicamentos concomitantes ao propranolol.

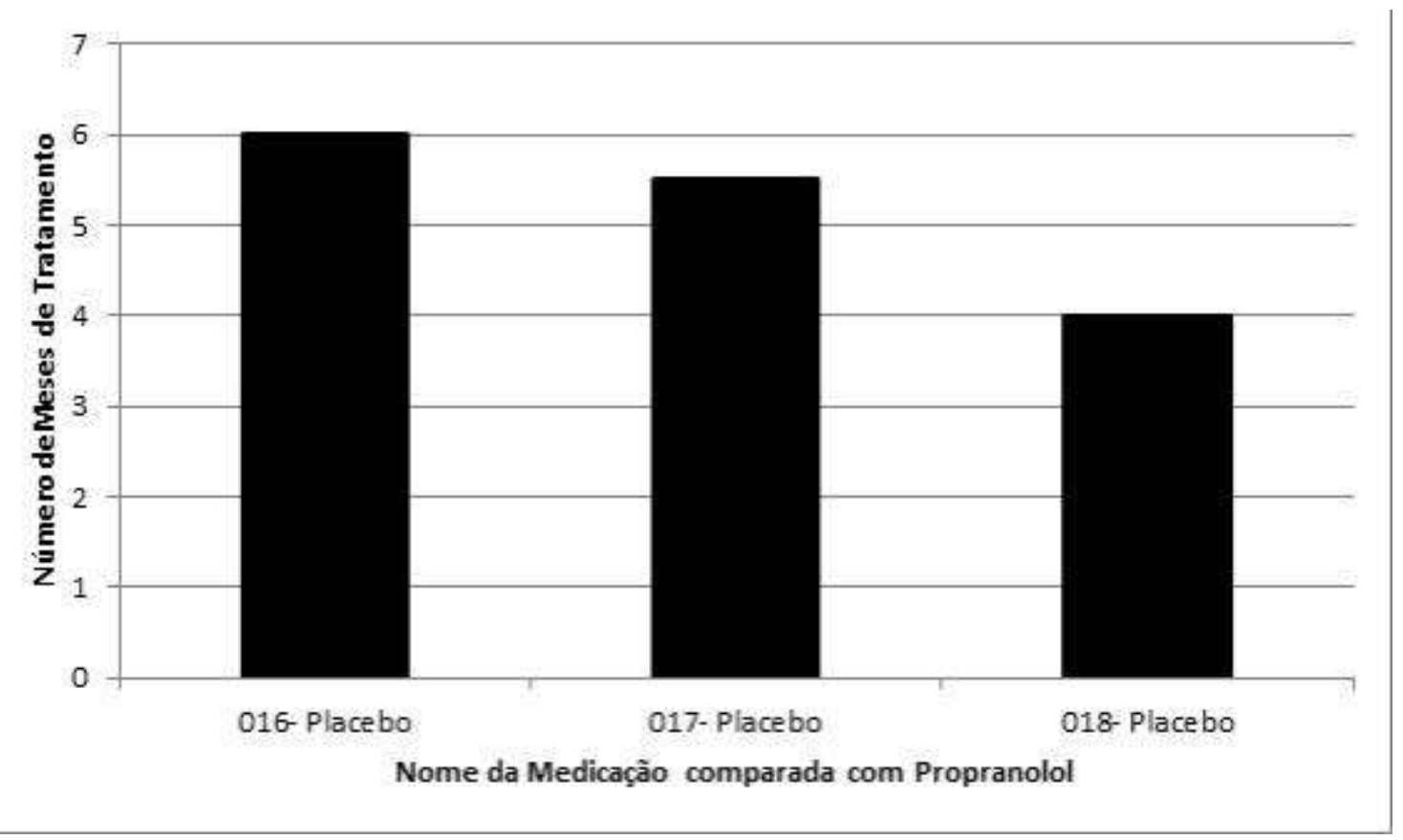

Fonte: Autores (2021).

\section{Discussão}

Os hemangiomas infantis são os tumores vasculares benignos mais comuns da infância. Existem vários fatores de risco, que incluem sexo feminino, prematuridade, baixo peso ao nascer, gestações múltiplas, idade materna avançada e fertilização in vitro. Mais de $60 \%$ de hemangiomas infantis afetam a região da cabeça e do pescoço. Morfologicamente, os hemangiomas são classificados em tipos superficiais, profundos e compostos de coloração avermelhada ou vinho. Quanto à terapêutica, o propranolol oral tornou-se o tratamento padrão para o hemangioma infantil profundo e de alto risco (Jing et al., 2016; Léauté-Labrèze et al., 2015; Baselga et al., 2018).

Os estudos 7, 14, 16 e 17 evidenciaram que o uso de Cloridrato de Propranolol em doses diárias de 3 mg/kg são eficazes na regressão do hemangioma infantil, sendo que no estudo 7 apenas 188 participantes de pesquisa cumpriram todo o tratamento no período de 24 semanas, enquanto no estudo 14 foi realizado pré teste que avaliou a função cardíaca de todos os participantes e os 31 participantes tiveram $100 \%$ de eficácia na proliferação do hemangioma e $87 \%$ de eficácia na involução do hemangioma, enquanto no estudo 16, que foi realizado de forma multicêntrica em dez hospitais, avaliou que $76 \%$ dos participantes obtiveram alta eficácia com o propranolol por um ano e que $68 \%$ mantiveram a eficácia pelo período de três meses após a finalização do estudo, sendo necessário que apenas $24 \%$ realizassem o ciclo terapêutico do início ou mudasse a terapia inicial (Léauté-Labrèze et al., 2015; Holmes et al., 2011; Baselga et al., 2018; Kaneko et al., 2017).

Já os estudos 1, 6, 11 e 18 mostraram que o tratamento utilizando $2 \mathrm{mg} / \mathrm{kg} /$ dia também se fez eficiente na redução do hemangioma infantil. No estudo 1 os hemangiomas involuiram completamente em casos simples na quarta semana de pesquisa, atingindo sua total eficácia na décima segunda semana em casos mais graves. Já no estudo 6, que retrata a terapia para hemangiomas nasais, houve uma redução de $61,5 \%$ dos casos, sendo necessário realizar uso do laser em $0,78 \%$ e cirurgias apenas em $1,38 \%$ da população total do estudo, enquanto o estudo 11 demonstrou que houve $80 \%$ da involução dos hemangiomas, porém após o término da terapia houve 3,31\% de reincidência, sendo necessário refazer a terapêutica. Em contrapartida, o estudo 18 mostrou-se diferente, mostrando que os participantes após a finalização do tratamento com o 
propranolol 5,26\% tiveram o retorno da doença, sendo necessário reiniciar o ciclo terapêutico ou fazer a troca da terapia escolhida (Hogeling et al., 2011; Perkins et al., 2014; Sondhi \& Patnaik, 2013; Hu et al., 2016).

O uso combinado de Cloridrato de Propranolol $2 \mathrm{mg} / \mathrm{kg} /$ dia e Maleato de Timolol 0,5\% foram apresentados como uma ótima opção no tratamento, uma vez que além de serem seguros, apresentaram excelentes resultados na involução do hemangioma infantil. O estudo de Holmes et al. (2011) mostrou uma redução de $100 \%$ nos hemangiomas em $87 \%$ dos participantes de pesquisa, e tendo um menor número de eventos adversos, enquanto estudo de Ge et al. (2016) demonstrou uma redução nos hemangiomas em $100 \%$ dos casos porém após os seis meses de terapia houve necessidade que $8,9 \%$ retomasse o tratamento do inicio devido a novas manifestações da doença.

Contudo, em outros estudos em que foi comparado a eficácia do Cloridrato de Propranolol e a Prednisolona, foi possível observar que houve uma resposta similar no uso de ambas as medicações. O estudo 2 se tratou de um estudo multicêntrico e demonstrou que a redução dos hemangiomas ocorreu em $41 \%$ dos casos no uso de prednisolona e de $64 \%$ dos casos de uso de propranolol, sendo que a prednisolona apresentou em 44 participantes eventos adversos graves em $44 \%$ dos casos, a terapia então teve seu tempo de estudo reduzido devido ao excesso de efeitos adversos. Sendo assim, o propranolol foi o único grupo que participou do estudo até o final, já no estudo 3 apresentaram uma redução nos hemangiomas em 100\% dos casos, porém após a finalização da terapia $8,9 \%$ necessitou de reinício terapêutico ou mudança de conduta terapêutica, enquanto no estudo 4 dois pacientes vieram a óbito devido a insuficiência cardíaca congestiva, insuficiência respiratória e coagulopatias secundário ao uso da prednisolona, demonstrando o risco do uso deste corticosteróide como terapêutica padrão, tornando assim o propranolol a nova terapia de escolha (Bauman et al., 2014; Ge, Zheng, Zhang, Yuan \& Zhao, 2016; Ji et al., 2016).

Já no estudo 12 o propranolol foi responsável pela maior taxa de eficácia mesmo em uso combinado com a prednisolona. No entanto, a prednisolona causava mais complicações, tendo assim baixa adesão dos participantes, enquanto no estudo 15 também demonstrou eventos adversos associadas a prednisolona, sendo que dois participantes desistiram do estudo e a eficácia do propranolol foi de 95,6\%, enquanto a da prednisolona foi de 91,9\%, com a ressalva que o propranolol não apresentou complicações. Sendo assim, o propranolol se mostrou superior, sendo a prednisolona responsável por maiores números de eventos adversos (Malik et al., 2013; Kim et al., 2017).

No estudo 8, com relação ao uso do Atenolol não houveram respostas que comprovasse a diferença ou superioridade com relação ao propranolol. Sendo assim acredita-se que ele possa entrar como opção no tratamento de hemangioma infantil. Durante o estudo observou-se que 53,8\% apresentaram respostas completas com o atenolol e 60\% com o uso do propranolol, mostrou também que nenhum paciente apresentou eventos adversos (Abarzúa-Araya et al., 2014).

No estudo 9, é possível observar que a combinação do Laser Pulsado de Corante Tópico (PDL) com o Cloridrato de Propranolol apresentou bastante eficácia e segurança, tendo uma resposta maior do que o mesmo isolado e apresentando ausência de efeitos colaterais. O estudo apresentou que entre os 10 pacientes que utilizaram PDL e propranolol tópico, 5 apresentaram excelentes respostas e os outros 5 apenas boas respostas. Apesar disso, acredita-se que seja necessário mais estudos controlados para a comprovação efetiva dos resultados (Ehsani et al., 2014).

Durante o estudo 15, a junção de Propranolol com Corticosteróides (Kenacort-A + Prednisolona + Celestone) no tratamento de Hemangioma Periocular (HOI) evidencia que apesar do propranolol ser a dose de primeira escolha, a injeção de corticoide ainda é uma alternativa segura para o tratamento de Hemangioma Periocular, sendo utilizada na ausência do propranolol. (Kim et al., 2017). 


\section{Considerações Finais}

Portanto, o hemangioma é o tumor benigno mais comum na infância, porém em alguns casos necessitam de tratamento para que involuam. Nesse sentido, o uso do propranolol é uma terapia alternativa para o tratamento de hemangioma infantil, podendo ser utilizado nas primeiras semanas de vida, após uma avaliação de condições cardíacas do recém nascido. O propranolol impede o crescimento do tumor e auxilia na diminuição da lesão. O mesmo apresenta alta eficácia e menos efeitos adversos do que o uso convencional de corticosteróides, laser pulsado de corante tópico, atenolol e timolol tópico. Sendo assim, o propranolol pode ser uma nova terapia de escolha para o hemangioma infantil. Sendo necessários mais estudos a fim de comprovar está hipótese.

\section{Referências}

Aly, M. M., Hamza, A. F., Kader, H. M. A., Saafan, H. A., Ghazy, M. S., \& Ragab, I. A. (2015). Superioridade terapêutica do propranolol combinado com curso curto de esteroides sobre a monoterapia com propranolol no hemangioma infantil. European journal of pediatrics, 174 (11), $1503-1509$.

Abarzúa-Araya, Á., Navarrete-Dechent, C. P., Heusser, F., Retamal, J., \& Zegpi-Trueba, M. S. (2014). Atenolol versus propranolol for the treatment of infantile hemangiomas: a randomized controlled study. Journal of the American Academy of Dermatology, 70(6), $1045-1049$.

Baselga, E., Dembowska-Baginska, B., Przewratil, P., González-Enseñat, M. A, Wyrzykowski, D., Torrelo, A., \& Voisard, JJ (2018). Eficácia do propranolol entre 6 e 12 meses de idade no hemangioma infantil de alto risco. Pediatrics, 142 (3).

Bauman, N. M., McCarter, R. J., Guzzetta, P. C., Shin, J. J., Oh, A. K., Preciado, D. A., \& Puttgen, K. B. (2014). Propranolol vs prednisolone for symptomatic proliferating infantile hemangiomas: a randomized clinical trial. JAMA Otolaryngology-Head \& Neck Surgery, 140(4), 323-330.

Brasil. (2018). Protocolo Clínico e Diretrizes Terapêticas Hemangioma Infantil. http://conitec.gov.br/images/Relatorios /2018/PCDT_Hemangioma_Infantil.pdf.

Buckmiller, L. M., Munson, P. D., Dyamenahalli, U., Dai, Y., \& Richter, G. T. (2010). Propranolol for infantile hemangiomas: early experience at a tertiary vascular anomalies center. The Laryngoscope, 120(4), 676-681.

Ehsani, AH, Noormohammadpoor, P., Abdolreza, M., Balighi, K., Arianian, Z., \& Daklan, S. (2014). Terapia combinada de hemangioma infantil com laser corante pulsado com propranolol tópico: um ensaio clínico randomizado. Arquivos da medicina iraniana, 17 (10), 0-0.

Ge, J., Zheng, J., Zhang, L., Yuan, W., \& Zhao, H. (2016). Propranolol oral combinado com timolol tópico para hemangiomas infantis compostos: um estudo retrospectivo. Relatórios científicos, 6 (1), 1-7.

Hogeling, M., Adams, S., \& Wargon, O. (2011). Um ensaio clínico randomizado de propranolol para hemangiomas infantis. Pediatrics, 128 (2), e259-e266.

Holmes, W. J. M., Mishra, A., Gorst, C., \& Liew, S. H. (2011). Propranolol as first-line treatment for rapidly proliferating infantile haemangiomas. Journal of plastic, reconstructive \& aesthetic surgery, 64(4), 445-451.

Hu, L., Zhou, B., Huang, H., Chang, L., Qiu, Y., Ma, G., ... \& Li, W. (2016). Efeitos do tratamento sistêmico com propranolol no crescimento físico de pacientes com hemangiomas infantis. The Journal of dermatology, 43 (10), 1160-1166.

Janmohamed, S. R., Madern, G. C., Nieuwenhuis, K., de Laat, P. C., \& Oranje, A. P. (2012). Evaluation of intra-lesional corticosteroids in the treatment of peri-ocular haemangioma of infancy: still an alternative besides propranolol. Pediatric surgery international, 28(4), 393-398.

Ji, Y., Chen, S., Xiang, B., Xu, Z., Jiang, X., Liu, X., ... \& Yang, L. (2016). Características clínicas e tratamento de hemangiomas hepáticos multifocais em crianças: um estudo retrospectivo. Relatórios científicos, 6 (1), 1-8.

CAMPOS JÚNIOR, D., Burns, D. A. R., \& Lopez, F. A. (2014). Tratado de pediatria.

Kaneko, T., Sasaki, S., Baba, N., Koh, K., Matsui, K., Ohjimi, H., ... \& Higuchi, T. (2017). Efficacy and safety of oral propranolol for infantile hemangioma in Japan. Pediatrics International, 59(8), 869-877.

Kim, K. H., Choi, T. H., Choi, Y., Park, Y. W., Hong, K. Y., Kim, D. Y., ... \& Jeong, J. H. (2017). Comparison of efficacy and safety between propranolol and steroid for infantile hemangioma: a randomized clinical trial. JAMA dermatology, 153(6), 529-536.

Kwon, EKM, Seefeldt, M., \& Drolet, BA (2013). Hemangiomas infantis. American Journal of Clinical Dermatology, 14 (2), $111-123$.

Léauté-Labrèze, C., Hoeger, P., Mazereeuw-Hautier, J., Guibaud, L., Baselga, E., Posiunas, G., ... \& Voisard, JJ (2015). Um ensaio clínico randomizado e controlado de propranolol oral em hemangioma infantil. $N$ Engl J Med, 372, 735-746.

Li, S. B., Xu, G. Q., Gao, F., \& Huo, R. (2015). Treatment with propranolol for infantile hemangiomas: A case series of 106 infants. Experimental and therapeutic medicine, 10(1), 51-54.

Malik, M. A., Menon, P., Rao, K. L. N., \& Samujh, R. (2013). Effect of propranolol vs prednisolone vs propranolol with prednisolone in the management of infantile hemangioma: a randomized controlled study. Journal of pediatric surgery, 48(12), 2453-2459. 
Research, Society and Development, v. 10, n. 8, e10010816968, 2021

(CC BY 4.0) | ISSN 2525-3409 | DOI: http://dx.doi.org/10.33448/rsd-v10i8.16968

Perkins, J. A., Chen, B. S., Saltzman, B., Manning, S. C., \& Parikh, S. R. (2014). Propranolol therapy for reducing the number of nasal infantile hemangioma invasive procedures. JAMA Otolaryngology-Head \& Neck Surgery, 140(3), 220-227.

Sondhi, V., \& Patnaik, SK (2013). Propranolol para hemangioma infantil (PINCH): um estudo aberto para avaliar a eficácia do propranolol no tratamento de hemangiomas infantis e para determinar o declínio da frequência cardíaca para prever a resposta ao propranolol. Journal of pediatric hematology / oncology, 35 (7), 493-499.

Souza, M. T. D., Silva, M. D. D., \& Carvalho, R. D. (2010). Revisão integrativa: o que é e como fazer. Einstein, 8(1), 102-106.

Szychta, P., Stewart, K., \& Anderson, W. (2014). Tratamento de hemangiomas infantis com propranolol: diretrizes clínicas. Cirurgia plástica e reconstrutiva, 133 (4), 852-862.

Tan, S. T, Itinteang, T., \& Leadbitter, P. (2011). Propranolol em baixa dosagem para hemangioma infantil. Journal of Plastic, Reconstructive \& Aesthetic Surgery, 64 (3), 292-299.

Wedgeworth, E., Glover, M., Irvine, A. D, Neri, I., Baselga, E., Clayton, T. H, ... \& Flohr, C. (2016). Propranolol no tratamento de hemangiomas infantis: lições da pesquisa do Grupo de Trabalho Europeu Propranolol no Tratamento de Haemangiomas Complicados (PITCH). British Journal of Dermatology, 174 (3), 594-601.

Yun, Y. J, Gyon, Y. H, Yang, S., Lee, Y. K, Park, J., \& Park, M. (2015). Um estudo prospectivo para avaliar a eficácia e segurança do propranolol oral como tratamento de primeira linha para hemangioma superficial infantil. Jornal coreano de pediatria, 58 (12), 484. 